(C) 2007 Elsevier Ltd. All rights reserved.

\footnotetext{
* Corresponding author. Tel.: +65 6790 6891; fax: +852 23625267.

E-mail address: ckmlee@inet.polyu.edu.hk (C.K.M. Lee).
}

\section{Introduction}

Today's enterprises require to face the challenge of responding to turbulent market change, meeting the escalating customer requirements and providing the qualitative, product within a short product lifecycle. Procurement is a crucial process and it accounts for more than half of enterprises' sales volume. For manufacturing firms, products are made of raw materials and components. For trading companies, purchasing the goods and then supplying the customers are the crucial business activities. As a result, procurement is regarded as a critical process in both manufacturing firms and trading companies. According to the

0957-4174/\$ - see front matter (C 2007 Elsevier Ltd. All rights reserved. doi:10.1016/j.eswa.2007.10.027

\title{
Design and development of agent-based procurement system to enhance business intelligence
}

\author{
C.K.M. Lee ${ }^{\mathrm{a}, *}$, H.C.W. Lau ${ }^{\mathrm{b}}$, G.T.S. Ho ${ }^{\mathrm{b}}$, William $\mathrm{Ho}^{\mathrm{c}}$ \\ ${ }^{a}$ Division of Systems and Engineering Management, School of Mechanical and Aerospace Engineering, Nanyang Technological University, \\ 50 Nanyang Avenue, Singapore 639798, Singapore \\ ${ }^{\mathrm{b}}$ Department of Industrial and Systems Engineering, The Hong Kong Polytechnic University, Hung Hom, Hong Kong \\ ${ }^{\mathrm{c}}$ Operations and Information Management Group, Aston Business School, Aston University, Birmingham B4 7ET, United Kingdom
}

\begin{abstract}
The purpose of this research is to propose a procurement system across other disciplines and retrieved information with relevant parties so as to have a better co-ordination between supply and demand sides. This paper demonstrates how to analyze the data with an agent-based procurement system (APS) to re-engineer and improve the existing procurement process. The intelligence agents take the responsibility of searching the potential suppliers, negotiation with the short-listed suppliers and evaluating the performance of suppliers based on the selection criteria with mathematical model. Manufacturing firms and trading companies spend more than half of their sales dollar in the purchase of raw material and components. Efficient data collection with high accuracy is one of the key success factors to generate quality procurement which is to purchasing right material at right quality from right suppliers. In general, the enterprises spend a significant amount of resources on data collection and storage, but too little on facilitating data analysis and sharing. To validate the feasibility of the approach, a case study on a manufacturing small and medium-sized enterprise (SME) has been conducted. APS supports the data and information analyzing technique to facilitate the decision making such that the agent can enhance the negotiation and suppler evaluation efficiency by saving time and cost.
\end{abstract}

Keywords: Case based reasoning; Procurement data processing; Price negotiation; OLAP; Agent and data management system

typical example illustrated by Arnold and Chapman (2004), profit can be increased by $10 \%$ either increasing sales volume by $10 \%$ or reducing the cost of purchase by $2 \%$. It is realized that that the efficient procurement practices can result in costly reduction, boost of profit and enhancing quality of the products. However, procurement is a complex process involving sourcing, analyzing, negotiating and assessing. A numerous problems have been identified in the past studies and shown below.

- Lack of co-ordination from buyer to production engineer.

- Lack of proactive and heavily depends on the request of production engineer.

- Paper-based purchasing cycle and lack of automation.

- Lack of intelligent advise tools provide for find out suitable suppliers. 
- Difficult to evaluate the performance of the suppliers.

The paper is organized to meet the following objectives to resolve the above problems:

- To present a procurement system across other disciplines and retrieved information with relevant parties.

- To illustrate how data are analyzed by OLAP.

- To propose the intelligent agent to facilitate a smooth procurement cycle.

- To demonstrate the case example about applying the proposed framework in electronic industry.

- To draw some conclusions and outline the needs for further research and development.

\section{Literature review}

A broad range of factors that can influence the efficiency of procurement has been mentioned in the literature. Most of the companies decentralize the operational control of their business units of functions geographically, which create different cultures, mission and constraints in different parties within the supply network. These cause difficulties to perform analysis and coordination, and prompt the need for a distributed information system to accelerate the information flow within the supply chain network. Information technologies, such as ERP system and agent-based system can be one of the solutions

Agent technology can be applied in various stage of purchasing cycle. APRON system, which provides product specification for procurement of construction products, automates the procurement process with seller agent and buyer agent. Apart form buying and selling, tracking the status of order is important for nowadays logistics operations. Trappey, Trappey, Hou, and Chen Bird (2004) proposed a mobile agent-based online logistics service tracking system (OLTSTS) for tracking the service status with agents at the dealer and data sides where the agent passes the input information to agent center to collect the information and answer the query responsively via web portal. OLSTS includes agent center as the service tracking kernel, the supply chain coordination channel and the global logistics service operation. Hadikusumo, Petchpong, and Charoenngam (2005) suggested to use electronic purchasing agent for searching, selecting the supplier and preparing purchase order. Lau et al. also proposed to deploy virtual agent for completing the assigned tasks in the form of "methods of objects". Virtual agents are supposed to carry out the designated tasks according to the breakdown procedures for inbound logistics (Lau, Wong, Pun, \& Chin, 2003). It should be noted that the various tasks undertaken by relevant agents could be carried out in geographicallydispersed companies in the supply chain network.

The assessment tools and evaluation criteria have been arisen for evaluating the performance of suppliers. Humphreys, Huang, and Cadden (2005) has proposed four types of indices to measure supplier involvement in design, namely: satisfaction index, flexibility index, risk index, and confidence index to measure the supplier capabilities and the potential or risk of signing a project contract. Davidrajuh (2003) also proposed to use quality, cost, delivery and critical performance measure in binary score (i.e. 1 for the critical factor is within the broad margin while 0 for the critical factor is within the broad margin) to select bidder.

Whilst there are many publications about applying agent technology in procurement process (Dzeng \& Lin, 2004; Lau et al., 2003; Valluri \& Croson, 2005), there is less literature about interconnecting the agent technology and OLAP in the whole purchasing cycle. In order to achieve responsiveness of the dynamic market, an agent-based procurement system (APS), which enables market data analysis and effective order processing, is proposed in this paper.

\section{Agent-based procurement system (APS)}

Agent-based procurement system consists of three major components which are data repository, business intelligence module (BIM) and procurement agents including search agent, negotiation agent and evaluation agent that has been shown in Fig. 1. Instead of having a pool of suppliers, more companies tend to have partnership with their major suppliers and order higher volume with their prime vendors. Data repository stores the corporate data and the shares data from suppliers and customer so as to let enterprise has a better collaborative purchasing practices. Business intelligence module makes use of OLAP to navigate and manipulate the market data interactively such that the buyers can determine the purchase quantity and realize the trend of the market. During the purchasing cycle, sourcing and engaging with suppliers and settling the transaction can be carried out by software agents.

\subsection{Data repository}

Data repository consists of database server or mainframe system to manage structured data, accept queries from users, and respond to those queries. Data repository has the following features (Sheldom, 1997):

(i) To provide a systematic method to store data as records, tables, or objects.

(ii) To enter the data and store the data for retrieval.

(iii) To provide query languages for searching, filtering, reporting, and other "decision-support" activities that help users to analysis the business environment and make decision with evidence.

(iv) To provide multi-user access to data, along with security features that prevent some users from viewing and changing certain types of information.

(v) To provide data integrity features that prevents more than one user from accessing and changing the same information simultaneously. 


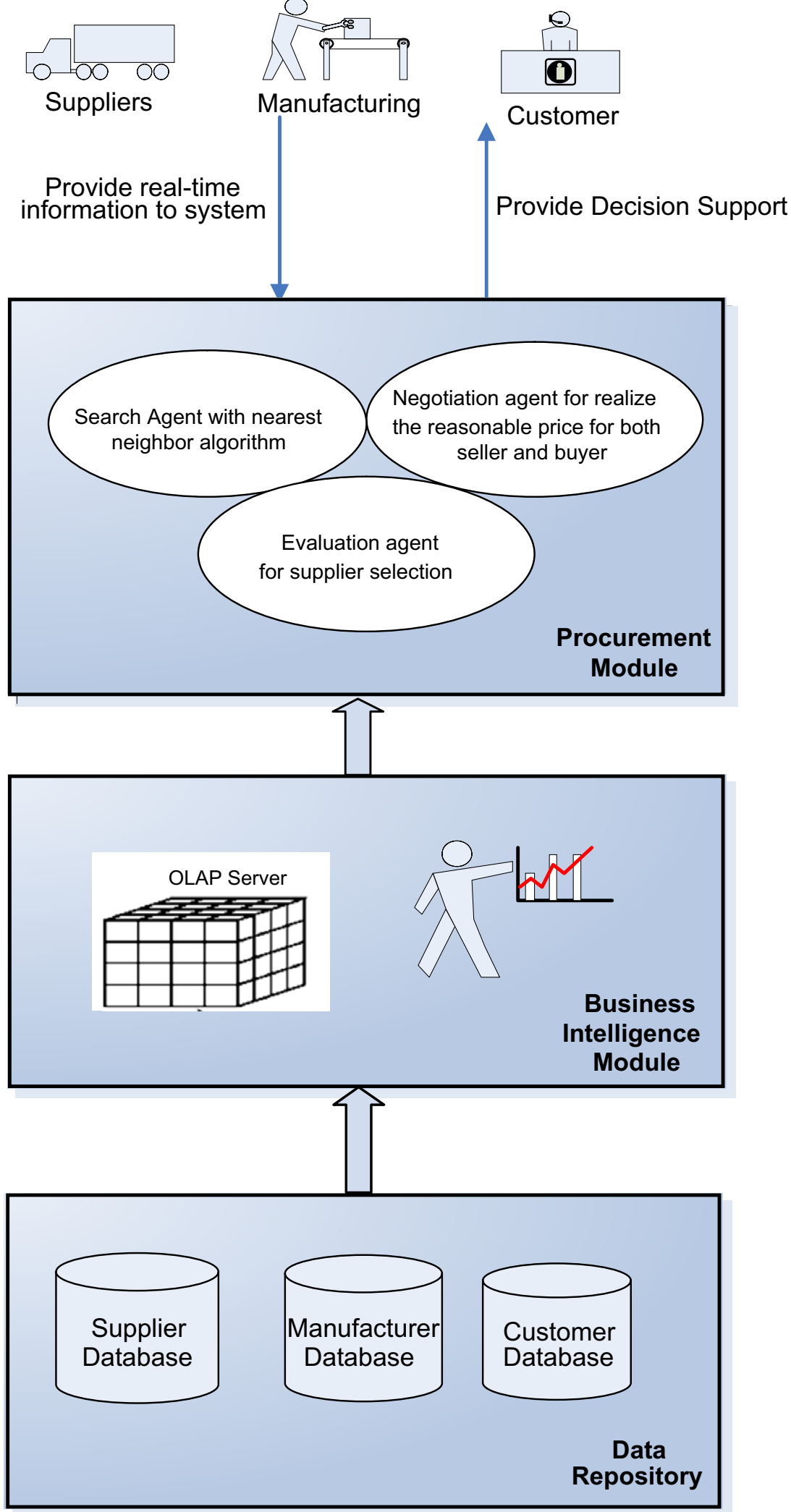

Fig. 1. Framework of agent-based procurement system.

(vi) To provide a data dictionary (metadata) that describes the structure of the database, related files, and record information.
According to Inmon (2002) who was the first one to raise the concept of data warehouse, data warehouse is a subject-oriented, integrated time-variant of data to support 
data analysis and decision making. Data mining which aims at discovering the hidden knowledge and crucial information among enormous amount of data can be viewed as a result of the natural evolution of information technology (Berson \& Smith, 1997; Michael \& Bel, 1999; Peterson, 2000; Robert, Joseph, \& David, 1999). On the other hand, according to Han and Kamber (2000), an evolutionary path has been witnessed that data mining is evolve from database management. In fact, it is realized that the new data repository is the computer-based software used to establish and manage various kinds of data structures such as data mart, transactional database, data warehouse. The overview of corporation data structures with linkages (Inmon, 2002) can be summarized in the data driven development methodology which can be applied in different industries.

\subsection{Business intelligence module}

Online analytical processing (OLAP) allows the decision makers to analysis data interactively (Datta \& Thomas, 1999). It leverages the time-variant characteristics of the data warehouse to allow the strategist to look up data in time-series. When examining the historical data, the analysts can identify hidden trends and clusters. The analyst can anticipate how the new policy affects the organization and top management can formulate the future strategy based on the historical information (Giovinazzo,
2002).The aim of OLAP is to give the decision makers a tool to detect the market trends and-analyze the characteristics of those trends (Gargano \& Raggad, 1999). OLAP allows users to view the data interactively and it has the capability to verifying or refuting the hypothesis. The OLAP allows the analysts to build models based on the data and manipulate the variables in the model so that the analyst can examine the business data into detail level or aggregated level (Giovinazzo, 2002).

Enterprise realizes that it is necessary to analyze and store the data in universal formats which can be better utilized for decision-making purposes. A more sophisticated technique is needed to cope with the explosion of data where OLAP is one of the tools to discover knowledge in the database.

OLAP tool is a set of business intelligence tools that allows the exploration - of data, creating and managing multidimensional enterprise data for analysis (Peterson, 2000; Thomsen, 1999). These are intended for non-analytical, non-technical users, allowing them to drill-down, sliceand-dice and pivoting the data. Two important concepts have to be borne in mind $\mathrm{OLAP}$ is application architecture, not intrinsically a data warehouse or a database management system (Berson \& Smith, 1997; Delvin, 1997) and OLAP is not data mining (Colet, 2003). In another word OLAP is set of functionalities that can facilitate multidimensional analysis and manipulate aggregated data into various categories shown in Fig. 2. Users can integrate

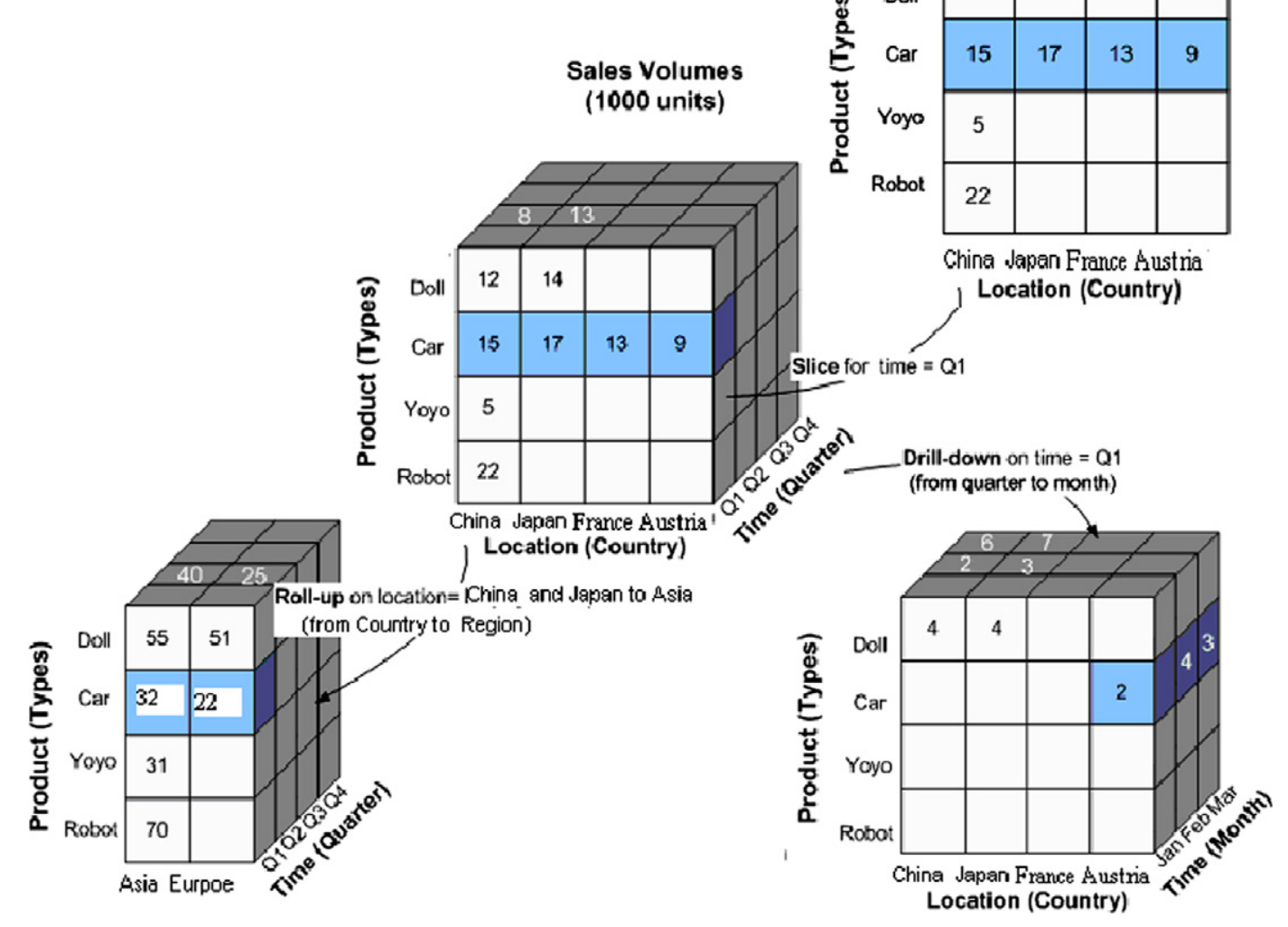

Fig. 2. Data analysis with OLAP. 
enterprise information through comparative customized view and analyze historical and projected data (Ma, Chou, \& Yen, 2000).

Data viewing across multiple dimensions is a key OLAP feature, The basic OLAP terminology is described as follows (Forsman, 1997; Inmon, 2002):

(i) Dimension: A category consistent group-of memberg represented as a specific axis of an OLAP cuba

(ii) Measures: a measure is the data in OLAP system. It is any quantitative expression. It is what is being analyzed across multiple dimensions.

(iii) Hierarchy: The organization of levels within a dimension that reflects (i) how addictive data is aggregated level by level and (ii) the top-down drill down path for users within the dimensiom

(iv) Member: The name or label for any member at any level in hierarchy ${ }_{\lambda}$ Bottom-level members are sometimes called leaf members.

(v) Family relationships: Terminology used for describing the relationships between members within a hierarchy.

(vi) Child: A member directly subordinate to another membenin hierarchy.

(vii) Parent: A member directly above another membenin a hierarchy.

(viii) Sibling: A member at the same level as one or more other members sharing the same parent.

(ix) Descendant: Any member at any lower level in relation to another specific member.

(x) Ancestor: Any member at any higher level in relation to another member.

\subsection{Procurement module}

Procurement module contains agents which possess social ability, proactiveness, reactivity and autonomy thereby enhancing the effectiveness of procurement practice with in the supply chain. Search agent encapsulates sociability such that it can enquire the bidding cost of potential suppliers. Instead of triggering the pre-written procedure in the program, agent is autonomous to accomplish the decomposed task to attain the goal. Most literatures may realize the importance of sourcing the suppliers and negotiating the better price with potential suppliers but ignoring the process after issuing the purchase order. After issuing the purchase order, buyers still need to follow up the delivery of the purchased components. The quality of incoming material needs to be checked by quality department and it is also a good way to evaluate the product quality of selected suppliers. Evaluation agent will retrieve the information such as defect rate, number of returns so as to determine to award another similar purchase order to the same supplier or not. Fig. 3 shows the purchasing cycle and the roles of procurement agents.

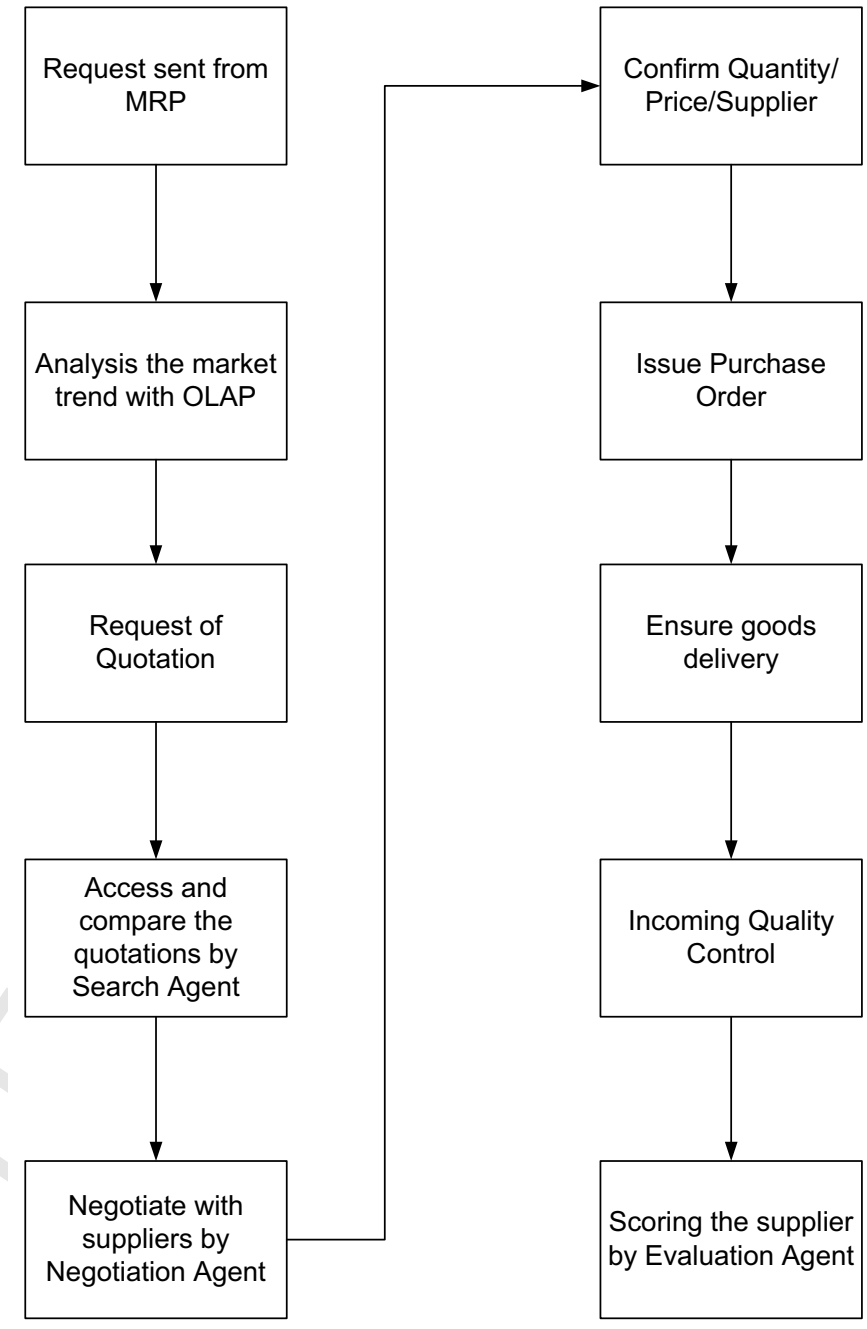

Fig. 3. Procurement process by APS.

\subsubsection{Search agent}

According to the findings of KPMG Consulting (2000), it is found that in the USA employees spend an average of $8 \mathrm{~h}_{\mathrm{\lambda}}$ each week retrieving external information. Agent based technology addresses the availability of information, the ability to exchange data seamlessly and the ability to process it across different applications in different organizational units. For repetitive orders, search agent retrieves the past purchase orders issued to the vendors and review the recent list price. For new order related to new products, engineers may design a technical specification and buyers may decide the payment term and logistics issues. Search agent will match and retrieve the potential suppliers prior to sorting a list of suppliers based on customers' specification. One of the methods to identify the similarity of cases is nearest neighbor algorithm (Koloder, 1993).

$\operatorname{similarity}($ CaseI, Case $)=\frac{\sum_{i=1}^{n} w_{i} \times \operatorname{sim}\left(f_{i}^{\mathrm{I}}, f_{i}^{\mathrm{R}}\right)}{\sum_{i=1}^{n} w_{i}}$ 
On receive accept from seller $\{$ exit; $\}$

\subsubsection{Evaluation agent}

Evaluation agent is to find out the performance of supplier based on delivery efficiency, reliability of quality, responsive to the market, cost and environmental friendly factor. Since global warming makes worldwide realize that the importance of environmental protection, international organization and government would like to advocate the green products and reduce the usage of hazardous components. Based on the equation proposed by Lau, Lee, Ho, Pun, and Choy (2006), this paper incorporates environmental protection factor which is one of the critical evaluation criteria of supplier selection. A prime vendor should maximize delivery efficiency $\left(D_{\max }\right)$, reliability of quality $\left(Q_{\max }\right)$ responsiveness to the market $\left(R_{\max }\right)$ and environment friendly ${ }_{\curlywedge}$ but to minimize the cost $\left(C_{\min }\right)$.

$$
\begin{aligned}
& F(x)=a D_{\max }+b Q_{\max }+c R_{\max }+d C_{\min }+e^{E} E C_{\min } \\
& D_{\max }=\sum_{i} \sum_{j} Y_{i j} \cdot\left(\frac{P_{i j}-D_{i j}-O_{i j}}{D_{i j}-P_{i j}}\right)
\end{aligned}
$$

$$
\begin{aligned}
Q_{\max } & =\sum_{i} \sum_{j} Y_{i j} \cdot A_{i j} \\
R_{\max } & =\sum_{i} \sum_{j} Y_{i j} \cdot S_{i j} \\
C_{\min } & =\sum_{i} \sum_{j} Y_{i j} \cdot U_{i j}+L_{i j} \\
E_{\max } & =\sum_{i} \sum_{j} \alpha \cdot G_{i j}-\beta \cdot H_{i j}
\end{aligned}
$$

Subject to:

$\sum_{j} Y_{i j}=K_{i}+F_{i}-M_{i}-N_{i} \forall i$

$Q_{i j \min } \geqslant Y_{i j} \forall i, j$,

$A_{i j} \geqslant 0.8 \forall i, j$,

$\frac{L_{i j}}{C_{i j}} \leqslant 0.1 \forall i, j$

$\frac{O_{i}}{P_{i j}-D_{i j}} \leqslant 0.1 \forall i$

The nomenclature is shown below:

$A_{i j} \quad$ acceptance rate of component $i$ from supplier $j$

$C_{i j} \quad$ total cost of component $i$ from supplier $j$

$D_{i j} \quad$ delivery date of component $i$ from supplier $j$

$E_{i j} \quad$ energy consumption of component $i$ from supplier $j$

$F_{i} \quad$ safety stock of component $i$

$G_{i j} \quad$ amount of component $i$ to be purchase from supplier $j$ which can be recycled and reuse

$H_{i j} \quad$ amount of component $i$ to be purchase from supplier $j$ which contain hazard substance or pollutant

$K_{i} \quad$ demand of component $i$

$L_{i j} \quad$ logistic cost of component $i$ from supplier $j$

$M_{i} \quad$ on hand inventory of component $i$

$N_{i} \quad$ on hand order of component $i$

$O_{i j} \quad$ order processing time of component $i$ from supplier $j$

$P_{i j} \quad$ purchase date of component $i$ from supplier $j$

$S_{i j} \quad$ score related to responsiveness of supplier $j$ for component $i$

$U_{i j} \quad$ unit cost of component $i$ from supplier $j$

$Y_{i j} \quad$ amount of component $i$ to be purchase from supplier $j$

$\alpha \quad$ reward factor for reusable and recyclable components provided by supplier

$\beta \quad$ penalty factor for components containing hazard substances and pollutant provided by supplier.

The constraints stated by Eqs. (7) and (8) are some considerations that need to be addressed in the procurement plan. The order quantity calculated by department of production and material control is the sum of material required and the safety stock minus the quantity of on hand inventory and on hand order. Buyers need to follow the minimum order quantity given by suppliers. Some constraints have been set in order to filter some suppliers who 


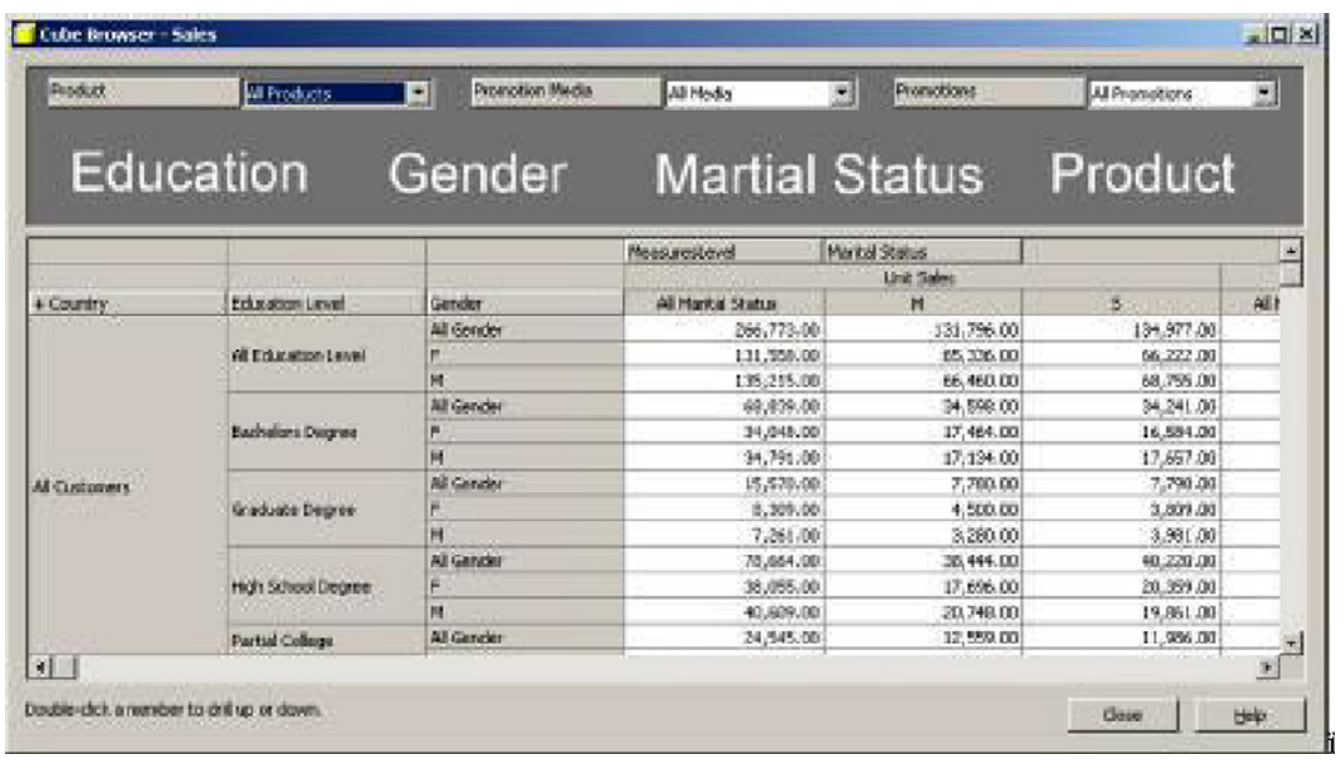

Fig. 4. OLAP data cube.

cannot meet the target of supply chain. For example, in Eq. (9) the acceptance rate of components received must be more 0.8 . The logistic cost should be less than or equal to $10 \%$ of the total cost (see Eq. (10)) and the order processing time should be less than one tenth of purchase lead time (see Eq. (11)).

\section{Illustrated case for APS}

KKS (aliased name) is an electrical appliance manufacturer which sold the products to European market. RoHS Directive, which stands for "the restriction of the use of certain hazardous substances in electrical and electronic equipment", came into force on 1 July 2006. This Directive bans the placing on the EU market of new electrical and electronic equipment containing more than agreed levels of lead, cadmium, mercury, hexavalent chromium, polybrominated biphenyl (PBB) and polybrominated diphenyl ether (PBDE) flame retardants. This directive may make electronic product manufactures re-design the products and reduce the usage of lead, cadmium which, in turn, reduce the procurement of those hazardous substance. Sharing the information related to bill of material can let suppliers know the amount of hazard substance of each IC components. Certain components with high level of hazard substance may be restricted and the purchase quantity will be decreased. That market information is entered to data repository and then manipulated by OLAP. To create an Excel PivotTable report based on an OLAP cube, Excel PivotTable Report Wizard is used and microsoft query applications are adopted to define and create an OLAP query file. The OLAP query file provides all the necessary information to connect the OLAP cube. When microsoft query returns control to the PivotTable Report Wizard, the wizard uses the OLAP query file to connect the cube. Fig. 4 shows the sales data of a particular electronic device of female graduate degree holder group.

That information provides a rough idea for buyers about the trend of the market. Procurement agent will be automatically triggered to find out the potential suppliers. Since the new purchasing order of electronic component need to compliance with RoHS, search agent will find out the suppliers with the capability to reach the quality standard. Negotiation agent may take extra care with the new negotiation terms such as payment term, price, order quantity, delivery date and agreement related to return of goods. Evaluation agent need to assess whether the components comply with new safety regulation. Even though the electronic components may pass the incoming quality control test, evaluation agent needs to keep track of the scrap rate from the shop floor so as to examine whether the components fit for the manufacturing process.

\section{Discussions and conclusions}

An agent-based procurement system is introduced in this paper. The proposed system aims to improve the current subjective practice of supplier selection, price negotiation and supplier evaluation by deploying the agent technology and OLAP. Although integrated systems have been reported in the literature previously, this study differs from the literature in terms of developing agent technology and deriving supplier evaluation function related to the procurement strategy of the company. This research is not only analyzing the market data and ensures quality procurement cycle in a systematic way, but also realizing the synergy of supplier collaboration so as to enhance the efficiency of the supply network. Search agent is used to find out the potential suppliers by matching the similarity of product specifications with offers given by suppliers. 
Negotiable agent engages in negotiation terms so as to achieve the mutual agreement. The derived supplier evaluation function consists of reliability of quality, delivery efficiency, competitiveness of the cost and the responsiveness to the market for evaluating the performance of supplier so as to have a better supplier management.

Further research will be focused on the refinement of the integrated agent based procurement system to achieve a more reliable and seamless integration with other ERP modules because procurement involves the intakes from other departments such as quality department and engineering department. Validation of the system should be implemented in the manufacturing firms such that feedback and comments of buyers and sellers helps to adjust the configuration of the system. The significant contribution of this paper is related to the effective introduction of agent technology and OLAP to the supply chain management and the dissertation of imparting intelligent demand pattern recognition to the procurement system. As designed, the proposed system enables the progressive intelligence features and elements into the supply chain. It is expected that the proposed system will enhance the international competitive edge of network manufacturers which are thereby enhancing the efficiency of the purchasing cycle.

\section{References}

Arnold, J. R. Tony, \& Chapman, Stephen N. (2004). Introduction to materials management. Upper Saddle River, NJ: Pearson/Prentice Hall.

Berson, A., \& Smith, S. (1997). Data warehousing, data mining \& OLAP (1st ed.). NY: McGraw Hill.

Colet, E., (2003). Looking at Data Mining Through a Portal. http:// www.hpcwire.com/dsstar/99/0302/100621.html.

Datta, A., \& Thomas, H. (1999). The cube data model: a conceptual model and algebra for online analytical processing in data warehouses. Decision Support Systems, 27(3), 289-301.

Davidrajuh, Reggie (2003). Modeling and implementation of supplier selection procedures for e-commerce initiatives. Industrial Management \& Data Systems, 103(1), 28-38.

Delvin, B. (1997). Data warehouse: From architecture to implementation. Reading, MA: Addison-Wesley.
Dzeng, Ren-Jye, \& Lin, Yu-Chun (2004). Intelligent agents for supporting construction procurement negotiation. Expert Systems with Applications, 27(1), 107-119.

Forsman, S. (1997). OLAP Council White Paper. OLAP Council. http:// www.olapcouncil.org/research/whtpaply.htm.

Gargano, Michael L., \& Raggad, Bel G. (1999). Data mining - A powerful information creating tool. OCLC System \& Services, 5(2), 81-90.

Giovinazzo, W. A. (2002). Internet-enabled business intelligence. USA: Prentice Hall.

Hadikusumo, B. H. W., Petchpong, Sataporn, \& Charoenngam, Chotchai (2005). Construction material procurement using Internet-based agent system. Automation in Construction, 14(6), 736-749.

Han, J., Kamber, M. (2000). Data Mining: Concepts and Techniques. Simon Fraser University, Intelligent Database Systems Research Laboratory, Canada.

Humphreys, Paul, Huang, George, \& Cadden, Trevor (2005). A webbased supplier evaluation tool for the product development process. Industrial Management \& Data Systems, 105(2), 147-163.

Inmon, W. H. (2002). Building the data warehouse (3rd ed.). NY: Wiley.

Koloder, J. L. (1993). Case-based reasoning. San Mateo, CA: Morgan Kaufman.

KPMG Consulting. (2000). Knowledge management research report, available at: ww.kmadvantage.com/docs $/ \mathrm{km}$ articles/KPMG_KM_ Research_Report_2000.pdf.

Lau, Henry C. W., Wong, Christina W. Y., Pun, K. F., \& Chin, K. S. (2003). Virtual agent modeling of an agile supply chain infrastructure. Journal of Management Decision, 41(7), 625-634.

Lau, H. C. W., Lee, C. K. M., Ho, G. T. S., Pun, K. F., \& Choy, K. L. (2006). A performance benchmarking system to support supplier selection. International Journal of Business Performance Management, $8(2), 132-151$.

Ma, C., Chou, D. C., \& Yen, D. C. (2000). Data warehousing, technology assessment and management. Industrial Management \& Data Systems, $100(3), 125-135$.

Michael, L. G., \& Bel, G. R. (1999). Data mining - a powerful information creating tool. OCLC Systems \& Services, 15(2), 15-24.

Peterson, T. (2000). Microsoft OLAP unleashed (2nd ed.). Indianapolis: Sams Pubishing.

Robert, S. C., Joseph, A. V., \& David, B. (1999). Microsoft data warehousing. John Wiley \& Sons.

Sheldom, T. (1997). The encyclopedia of networking and telecommunications (2nd ed.). NY: Mc Graw Hill.

Thomsen, E. (1999). Microsoft OLAP solutions. New York: J. Wiley.

Trappey, Amy J. C., Trappey, Charles V., Hou, Jiang-Liang, \& Chen Bird, J. G. (2004). Mobile agent technology and application for online global logistic services. Industrial Management \& Data Systems, 104(2), $169-183$.

Valluri, Annapurna, \& Croson, David C. (2005). Agent learning in supplier selection models. Decision Support Systems, 39(2), 219-240. 


\section{University Library}

\section{- M M N E R VA A gateway to Melbourne's research publications}

Minerva Access is the Institutional Repository of The University of Melbourne

Author/s:

Lee, CKM;Lau, HCW;Ho, GTS;Ho, W

Title:

Design and development of agent-based procurement system to enhance business intelligence

Date:

2009-01-01

Citation:

Lee, C. K. M., Lau, H. C. W., Ho, G. T. S. \& Ho, W. (2009). Design and development of agent-based procurement system to enhance business intelligence. EXPERT SYSTEMS WITH APPLICATIONS, 36 (1), pp.877-884. https://doi.org/10.1016/j.eswa.2007.10.027.

Persistent Link:

http://hdl.handle.net/11343/118671 